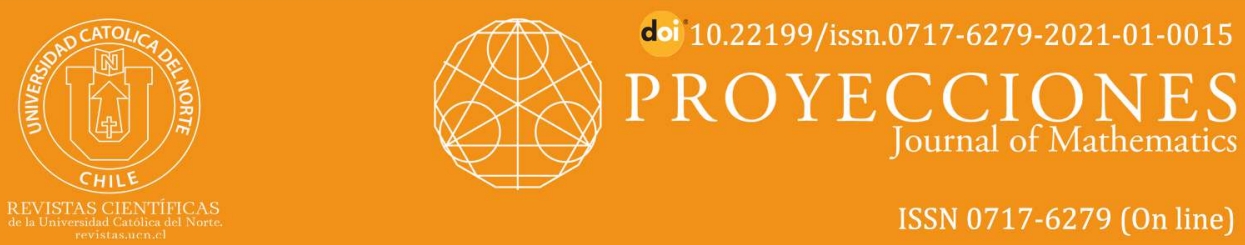

\title{
On a bi-nonlocal fourth order elliptic problem
}

F. Jaafri ${ }^{1}$

A. Ayoujil ${ }^{2}$ (i) orcid.org/0000-0002-0559-3242

M. Berrajaa ${ }^{3}$

${ }^{1}$ University Mohamed I, Dept. of Mathematics, LAMAO, Oujda, Morocco.

\aafri.fatna.sma@gmail.com

${ }^{2}$ Regional Centre of Trades Education and Training, Oujda, Morocco.

abayoujil@gmail.com

${ }^{3}$ University Mohamed I, Dept. of Mathematics, Oujda, Morocco.

berrajaamo@yahoo.fr

Received: August 2020 | Accepted: September 2020

\section{Abstract:}

This paper is aiming at obtaining weak solution for a bi-nonlocal fourth order elliptic problem with Navier boundary condition. Our approach is based on variational methods and critical point theory.

Keywords: Bi-nonlocal elliptic problem; $p(x)$-biharmonic operator; Mountain pass lemma; Variational method; Critical point theory; Navier boundary condition.

MSC (2020): 05C50, 05C82, 05C90.

\section{Cite this article as (IEEE citation style):}

F. Jaafri, A. Ayoujil, and M. Berrajaa, "On a bi-nonlocal fourth order elliptic problem", Proyecciones (Antofagasta, On line), vol. 40, no. 1, pp. 239-253, 2021, doi: 10.22199/issn.07176279-2021-01-0015

Article copyright: (C) 2021 F. Jaafri, A. Ayoujil, and M. Berrajaa. This is an open access article distributed under the terms of the Creative Commons License, which permits unrestricted use and distribution provided the original author and source are credited. 


\section{Introduction and main results}

In recent years, a great deal of attention has been paid to the study of problems involving nonlocaloperators, both in the pure mathematical research and in the concrete real-world applications, such as, optimization, finance, continuum mechanics, phase transition phenomena, population dynamics, and game theory, see $[7,8,6]$ and references therein.

In this paper, we are interested in the existence of weak solutions for the following fourth order elliptic equations of Kirchhoff type, with an additional nonlocal term,

$$
\left\{\begin{array}{c}
M\left(\int_{\Omega} \frac{1}{p(x)}|\Delta u|^{p(x)} d x\right) \Delta_{p(x)}^{2} u=\lambda|u|^{q(x)-2} u\left[\int_{\Omega} \frac{1}{q(x)}|u|^{q(x)} d x\right]^{r} \text { in } \Omega, \\
\Delta u=u=0
\end{array}\right.
$$

where $\Omega \subset \mathbf{R}^{N}(N \geq 1)$ is bounded smooth domain, $\Delta_{p(x)}^{2} u=\Delta\left(|\Delta u|^{p(x)-2} \Delta u\right)$ is the $p(x)$-biharmonic operator, $\lambda, r>0$ are real parameters and $p, q \in$ $C_{+}(\bar{\Omega})=\{h: h \in C(\Omega) ; h(x)>1, \forall x \in \bar{\Omega}\}$. Here $M:[0,+\infty) \rightarrow \mathbf{R}$ is a continuous function satisfying condition which will be stated later.

Since the two equation in (1.1) contains an integral over $\Omega$, it is no longer a pointwise identity; therefore it is often called bi-nonlocal problem.

$$
\int_{\Omega} \frac{1}{p(x)}|\Delta u|^{p(x)} d x \text { and } \int_{\Omega} \frac{1}{q(x)}|u|^{q(x)} d x .
$$

As it is well known, the interest of such problems comes from the fact that Kirchhoff type problems usually model several physical and biological systems, where $u$ describes a process which depends on the average of itself, such as the population density. Moreover, problems like (1.1) is related to the stationary version of a model, the so-called Kirchhoff equation, introduced by Kirchhoff in 1883 (see [11]). To be more precise, Kirchhoff established a model given by the equation

$$
\rho \frac{\partial^{2} u}{\partial t^{2}}-\left(\frac{P_{0}}{h}+\frac{E}{2 L} \int_{0}^{L}\left|\frac{\partial u}{\partial x}\right|^{2}\right) \frac{\partial^{2} u}{\partial x^{2}}=0,
$$

where $\rho, P_{0}, h, E, L$ are constants, which extends the classical D'Alambert's wave equation, by considering the effects of the changes in the length of the strings during the vibrations.

In recent years, there has been increasing attention to study a problem like (1.1) because such equations can describe some phenomena appeared in 
physics and engineering, such as describing the theorem of beam vibration, image processing, and so on.

In [8], the authors studied the following Kirchhoff equation

$$
\left\{\begin{array}{c}
-M\left(\int_{\Omega} \frac{1}{p(x)}|\nabla u|^{p(x)} d x\right) \Delta_{p(x)} u=\lambda|u|^{q(x)-2} u\left[\int_{\Omega} \frac{1}{q(x)}|u|^{q(x)} d x\right]^{r} \text { in } \Omega \\
u=0
\end{array}\right.
$$

By using variational methods, they proved several results on the existence of positive solutions.

In the [7], the authors study the existence and multiplicity of solutions via Krasnoselskii's Genus, to the following bi-nonlocal $\mathrm{p}(\mathrm{x})$-Kirchhoff equation,

$\left\{\begin{array}{cc}-M\left(\int_{\Omega} \frac{1}{p(x)}|\nabla u|^{p(x)} d x\right) \Delta_{p(x)} u=f(x, u)\left[\int_{\Omega} F(x, u) d x\right]^{r} & \text { in } \Omega, \\ u=0 & \text { on } \partial \Omega,\end{array}\right.$

with $F(t)=\int_{0}^{t} f(s) d s$, where $f: \mathbf{R} \rightarrow \mathbf{R}$ is a given regular function.

The $p(x)$-biharmonic problem is the general form of the $p$-biharmonic problem. The operator is no longer a satisfied homogeneous and pointwise identity. Many authors have studied the following problem (see for example $[1],[2],[3],[4],[5])$

$$
\left\{\begin{array}{cc}
\Delta\left(|\Delta u|^{p(x)-2} \Delta u\right)=f(x, u) & \text { in } \Omega \\
u=\Delta u=0 & \text { on } \partial \Omega
\end{array}\right.
$$

In [1], when $f(x, u)=\lambda|u|^{q(x)-2} u$, the authors proved the existence of many eigenvalue sequences via Ljusternik-Schnirelmann theory on $C^{1}$-manifolds. In [2], through the mountain pass lemma and Ekelands variational principale and under a suitable conditions, they established the existence of a continuous family of eigenvalues. When $f(x, u)=\lambda V(x)|u|^{q(x)-2} u$ in problem (1.4), many existence and nonexistence results are obtained $[4,5]$.

In the literature, biharmonic equations with a nonlocal term have received more attention and have been the subject of extensive study, we refer to $[13,14,15,16,17]$ for more detail. To the best of our knowledge, the 
study of elliptic problem with bi-nonlocal terms of the form (1.1) possesses more complicated because there is no maximum principle for the biharmonic problem and the combined effects of the bi-nonlocal terms provoke some mathematical dificulties. As far as we are aware, bi-nonlocal problems like (1.1), have not yet been studied. That is why, at our best knowledge, the present paper is a first contribution in this direction.

Motivated by the above works papers, the chief aim of this article is to research the system (1.1) under appropriate conditions.

Let us define, for every $x \in \Omega$,

$$
p^{*}(x)= \begin{cases}\frac{N p(x)}{N-2 p(x)} & p(x)<\frac{N}{2} \\ +\infty & p(x) \frac{N}{2} .\end{cases}
$$

Throughout the paper, we will assume that:

(H) $1 \leq p(x) \leq \frac{N}{2}$ with $1<q(x)<p^{*}(x)$ for all $x \in \bar{\Omega}$,

(M) There exist $m_{1} \geq m_{0}>0$ such that for all $t \in \mathbf{R}^{+}, m_{0} \leq M(t) \leq m_{1}$.

Here, problem (1.1) is stated in the framework of the generalized Sobolev space

$$
X=\left\{W^{2, p(x)}(\Omega): \quad u=0 \text { and } \Delta u=0 \text { in } \partial \Omega\right\}
$$

for witch some stated elementary properties are stated below.

By a weak solution to problem (1.1) we understand a function $u \in X \backslash\{0\}$ such that

$$
\begin{aligned}
& M\left(\int_{\Omega} \frac{1}{p(x)}|\Delta u|^{p(x)} d x\right) \int_{\Omega}|\Delta u|^{p(x)-2} \Delta u \Delta v d x \\
= & \lambda\left[\int_{\Omega} \frac{1}{q(x)}|u|^{q(x)} d x\right]^{r} \int_{\Omega}|u|^{q(x)-2} u v d x, \quad \forall v \in X .
\end{aligned}
$$

The energy functional corresponding to problem eq:Robin is defined as $J_{\lambda}: X \rightarrow \mathbf{R}$

$$
J_{\lambda}(u)=\tilde{M}\left(\int_{\Omega} \frac{1}{p(x)}|\Delta u|^{p(x)} d x\right)-\frac{\lambda}{r+1}\left[\int_{\Omega} \frac{1}{q(x)}|u|^{q(x)} d x\right]^{r+1},
$$

where $\tilde{M}(t)=\int_{0}^{t} M(s) d s$.

It is well known, $J_{\lambda} \in C^{1}(X, \mathbf{R})$ and

$$
J_{\lambda}^{\prime}(u)(v)=M\left(\int_{\Omega} \frac{1}{p(x)}|\Delta u|^{p(x)} d x\right) \int_{\Omega}|\Delta u|^{p(x)-2} \Delta u \Delta v d x
$$




$$
-\lambda\left[\int_{\Omega} \frac{1}{q(x)}|u|^{q(x)} d x\right]^{r} \int_{\Omega}|u|^{q(x)-2} u v d x
$$

for all $u, v \in X$.

Thus, the weak solution of problem (1.1), coïncide with the critical point of $J_{\lambda}$.

Define the mappings $T, G: X \rightarrow X^{\prime}$ by

$$
T(u)=\tilde{M}(u) L_{p(x)}(u)
$$

and

$$
G(u)=B(u) N(u)
$$

where

$\left\langle L_{p(x)}(u), v\right\rangle=\int_{\Omega}|\Delta u|^{p(x)-2} \Delta u \Delta v d x \forall u, v \in X, B(u)=\left[\int_{\Omega} \frac{1}{q(x)}|u|^{q(x)} d x\right]^{r}$

and

$$
\langle N(u), v\rangle=\int_{\Omega}|u|^{q(x)-2} u v d x \quad \forall u, v \in X .
$$

We can write $J_{\lambda}$ as

$$
J_{\lambda}(u)=T(u)-\lambda G(u) .
$$

Now, we are ready to give the main results of our paper.

Theorem 1.1. Under the conditions $(H)$ and $(M)$. In addition, assume that $\frac{m_{1} p^{+}}{m_{0}}<\frac{\left(q^{-}\right)^{(r+1)}(r+1)}{\left(q^{+}\right)^{r}}$ and $p^{+}<q^{-}(r+1)$. Then, for all $\lambda>0$, problem (1.1) possesses a nontrivial weak solution.

Theorem 1.2. Under the conditions $(H)$ and $(M)$. If $p^{-}>q^{+}(r+1)$., then, for any $\lambda>0$, there exists a sequence $\left(u_{n}\right)$ of non trivial weak solutions for problem (1.1) such that $u_{n} \rightarrow 0$ in $X$.

The remainder of this paper is organized as follows. The section 2 countains some preliminary properties concerning the generalized LebegueSobolev space and embedding result. The proofs of main results are given in section 3 . 


\section{Preliminaries}

In this section, we first recall some basic results, which will be used in the next section.

Firstly, we introduce some theories of Lebegue-Sobolev spaces with variable exponent. For details, see $[9,10]$. Define

$$
\forall h \in C_{+}(\bar{\Omega}), h^{-}=\min _{x \in \bar{\Omega}} h(x) \leq h^{+}=\max _{x \in \bar{\Omega}} h(x) .
$$

For $p \in C_{+}(\bar{\Omega})$, we define the variable exponent Lebegue space

$$
L^{p(x)}(\Omega)=\left\{u: \Omega \rightarrow \mathbf{R} \text { mesurable; } \int_{\Omega}|u(x)|^{p(x)} d x<\infty\right\},
$$

with the norm

$$
|u|_{p(x)}=\inf \left\{\mu>0: \int_{\Omega}\left|\frac{u}{\mu}\right|^{p(x)} d x \leq 1\right\}
$$

and it is a separable and reflexive Banach space.

Proposition 2.1. ([9]) For $u \in L^{p(x)}(\Omega)$ and $v \in L^{q(x)}(\Omega)$, we have

$$
\left|\int_{\Omega} u v d x\right| \leq\left(\frac{1}{p^{-}}+\frac{1}{q^{-}}\right)|u|_{p(x)}|v|_{q(x)}
$$

where $\frac{1}{p(x)}+\frac{1}{q(x)}=1$.

Proposition 2.2. ([9]) Let $\rho(u)=\int_{\Omega}|u|^{p(x)} d x$. For $u, u_{n} \in L^{p(x)}(\Omega)$, we have

1. $|u|_{p(x)}<1(\operatorname{resp}=1,>1) \Leftrightarrow \rho(u)<1(\operatorname{resp}=1,>1)$.

2. $\min \left(|u|_{p(x)}^{p^{-}},|u|_{p(x)}^{p^{+}}\right) \rho(u) \max \left(|u|_{p(x)}^{p^{-}},|u|_{p(x)}^{p^{+}}\right)$.

3. $\left|u_{n}(x)\right|_{p(x)} \rightarrow 0(\operatorname{resp} \rightarrow \infty) \Leftrightarrow \rho\left(u_{n}\right) \rightarrow 0(\operatorname{resp} \rightarrow \infty)$.

Define the variable exponent Sobolev space, for any positive integer $k$, set

$$
W^{k, p(x)}(\Omega)=\left\{u \in L^{p(x)}(\Omega): \quad D^{\alpha} u \in L^{p(x)}(\Omega), \quad|\alpha| \leq k\right\},
$$


where $\alpha=\left(\alpha_{1}, \alpha_{2}, \ldots . ., \alpha_{N}\right)$ is a multi-index, $|\alpha|=\sum_{i=1}^{N} \alpha_{i}$ and $D^{\alpha} u=\frac{\partial^{|\alpha|} u}{\partial x_{1}^{\alpha} \ldots \ldots \partial x_{N}^{\alpha_{N}}}$,

with the norm

$$
\|u\|_{k, p(x)}=\sum_{|\alpha| \leq k}\left|D^{\alpha} u\right|_{p(x)} .
$$

Then, $W^{k, p(x)}(\Omega)$ also becomes a seperable, reflexive and Banach space. We denote by $W_{0}^{k, p(x)}(\Omega)$ the closure of $C_{0}^{\infty}(\Omega)$ in $W^{k, p(x)}(\Omega)$.

Definition 2.3. Assume that spaces $E, F$ are Banach spaces, we define the norm on the space $E \cap F$ as $\|u\|=\|u\|_{E}+\|u\|_{F}$.

From the above definition, we can know that for any $u \in X,\|u\|_{X}=\|$ $u\left\|_{1, p(x)}+\right\| u \|_{2, p(x)}$, thus $\|u\|_{X}=|u|_{p(x)}+|\nabla u|_{p(x)}+\sum_{|\alpha|=2}\left|D^{\alpha} u\right|_{p(x)}$.

In Zanga and $\mathrm{Fu}$ [18], the equivalence of the norms was proved, and it was even proved that the norm $|\Delta u(x)|_{p(x)}$ is equivalent to the norm $\|u\|_{X}$ (see [[18], Theorem 4.4]). Let us choose on $X$ the norm defined by

$$
\|u\|=|\Delta u(x)|_{p(x)} .
$$

Note that, $(X,\|\cdot\|)$ is also a separable and reflexive Banach space. Similar to Proposition 2.1, we have the following

Proposition 2.4 (1). Let $I(u)=\int_{\Omega}|\Delta u|^{p(x)} d x$. For $u, u_{n} \in L^{p(x)}(\Omega)$, we have

1. $\|u\|<1(\operatorname{resp}=1,>1) \Leftrightarrow I(u)<1(\operatorname{resp}=1,>1)$.

2. $\min \left(\|u\|^{p^{-}},\|u\|^{p^{+}}\right) I(u) \max \left(\|u\|^{p^{-}},\|u\|^{p^{+}}\right)$.

3. $\left\|u_{n}-u\right\| \rightarrow 0 \Leftrightarrow I\left(u_{n}-u\right) \rightarrow 0$.

Remark 2.1 Let $h \in C_{+}(\bar{\Omega})$ and $h(x)<p^{*}(x)$ for any $x \in \bar{\Omega}$. Then, by [[1], Theorem 3.2], we deduce that $X$, is continuously and compactly embedded in $L^{h(x)}(\Omega)$. 
Proposition 2.5 (2). $\quad$ i) $-N$ is completly continuous, namely, $u_{n} \rightarrow u$ in $X$ implies $N\left(u_{n}\right) \rightarrow N(u)$ in $X^{\prime}$.

ii)- $L_{p(x)}$ satisfies condition $\left(S_{+}\right)$, namely, $u_{n} \rightarrow u$ and $\limsup \left\langle L_{p(x)}\left(u_{n}\right), u_{n}-\right.$ $u\rangle \leq 0$, imply $u_{n} \rightarrow u$ in $X$.

We need the following Symmetric Mountain Pass lemma to prove theorem 1.2.

Theorem 2.6. ([12]) Let $X$ be an infinite dimentional Banach space and $I \in C^{1}(X, \mathbf{R})$ satisfy the following two assumptions:

(A1) $I(u)$ is even, bounded from below. $I(0)=0$ and $I(u)$ satisfies the Palais Smale condition $(P S)$, namely, any sequence $u_{n}$ in $E$ such that $I\left(u_{n}\right)$ is bounded and $I^{\prime}\left(u_{n}\right) \rightarrow 0$ as $n \rightarrow \infty$ has a convergent subsequence.

(A2) For each $k \in \mathbf{N}$, there exists an $A_{k} \in \Gamma_{k}$ such that $\sup _{u \in A_{k}} J(u)<0$.

Then, $I(u)$ admits a sequence of critical points $u_{k}$ such that

$$
I\left(u_{k}\right)<0, \quad u_{k} \neq 0 \text { and } \lim _{k} u_{k}=0
$$

where $\Gamma_{k}$ denote the family of closed symmetric subsets $A$ of $E$ such that $0 \notin A$ and $\gamma(A) \geq k$ with $\gamma(A)$ is the genus of $A$, i.e

$\gamma(K)=\inf \left\{k \in N: \exists h: K \rightarrow \mathbf{R}^{k} \backslash\{0\}\right.$ such that $h$ is continuous and odd $\}$.

\section{Proof of Theorems 1.1 and $\mathbf{1 . 2}$}

\section{Proof of Theorem 1.1.}

For applying the Mountain Pass Theorem. We start with the following lemmas.

Lemma 3.1. There exist $\rho, C^{\prime}>0$ such that $J_{\lambda}(u) \geq C^{\prime}$ for all $u \in X$ such that $\|u\|=\rho$. 
Proof. Recalling that

$$
J_{\lambda}(u) \geq \frac{m_{0}}{p^{+}} \int_{\Omega}|\Delta u|^{p(x)} d x-\frac{\lambda}{r+1}\left[\int_{\Omega} \frac{1}{q(x)}|u|^{q(x)} d x\right]^{r+1} .
$$

In other hand, since $q(x)<p^{*}(x)$ for all $x \in \bar{\Omega}$, it follows that $X$ in continuous embedded in $L^{q(x)}(\Omega)$. So, there exists a positive constant $C_{1}$ such that

$$
|u|_{q(x)} \leq C_{1}\|u\|, \quad \forall u \in X .
$$

Let us fix $\rho \in(0,1)$ such that $\rho<\frac{1}{C_{1}}$. Then relation $\mathrm{p} 4$ implies $|u|_{q(x)}<$ 1 , for all $u \in X$ with $\|u\|=\rho$.

By proposition (2.2) and immersions Sobolev, we have

$$
\int_{\Omega}|u|^{q(x)} \leq|u|_{q(x)}^{q^{-}} \leq C_{1}^{q^{-}}\|u\|^{q^{-}}, \quad \forall u \in X
$$

it follows that,

$$
\begin{aligned}
J_{\lambda}(u) & \geq \frac{m_{0}}{p^{+}} \rho^{p^{+}}-\frac{\lambda}{(r+1)\left(q^{-}\right)^{r+1}} C_{1}^{q^{-}(r+1)} \rho^{q^{-}(r+1)} \\
& \geq \rho^{p^{+}}\left(\frac{m_{0}}{p^{+}}-\frac{\lambda}{(r+1)\left(q^{-}\right)^{(r+1)}} C \rho^{q^{-}(r+1)-p^{+}}\right) .
\end{aligned}
$$

Since $q^{-}(r+1)>p^{+}$, the function $\rho \rightarrow \frac{m_{0}}{p^{+}}-\frac{\lambda}{(r+1)\left(q^{-}\right)^{(r+1)}} C \rho^{q^{-}(r+1)-p^{+}}$ strictly positive an neighborhood of the origin. So, we find positive numbers $\rho, C^{\prime}$ such that

$$
J_{\lambda}(u) \geq C^{\prime}, \quad\|u\|=\rho .
$$

Lemma 3.2. There exists $e \in X$ with $\|e\| \geq \rho$ such that $J_{\lambda}(e)<0$, where $\rho$ is given in lemma 3.1

Proof. Choose $\psi \in C_{0}^{\infty}, \psi \neq 0$. For $t>1$, we have

$$
J_{\lambda}(t \psi) \leq \frac{m_{1} t^{p^{+}}}{p^{-}} \int_{\Omega}|\Delta \psi|^{p(x)} d x-\frac{\lambda}{r+1} \frac{t^{q^{-}(r+1)}}{\left(q^{+}\right)^{r+1}}\left(\int_{\Omega}|\psi|^{q(x)} d x\right)^{r+1} .
$$

Using the fact that $q^{-}(r+1)>p^{+}$, we obtain $J_{\lambda}(t \psi) \rightarrow-\infty$ as $t \rightarrow+\infty$. Therefore, for $t>1$ large enought, there is $e=t \psi$ such that $\|e\| \geq \rho$ and $J_{\lambda}(e)<0$. This completes the proof.

Lemma 3.3. The functional $J_{\lambda}$ satisfies the condition $(P S)$. 
Proof. Let $\left(u_{n}\right) \subset X$ be a sequence such that,

$$
J_{\lambda}\left(u_{n}\right) \rightarrow d \text { and } J_{\lambda}^{\prime}\left(u_{n}\right) \rightarrow 0 \text { in } X^{\prime} .
$$

By contradiction suppose that

$$
\left\|u_{n}\right\| \rightarrow+\infty \text { as } n \rightarrow \infty \text { and }\left\|u_{n}\right\|>1 \text { for any } n \text {. }
$$

Thus, taking $\frac{m_{1} p^{+}}{m_{0}}<\theta<\frac{\left(q^{-}\right)^{r+1}(r+1)}{\left(q^{+}\right)^{r}}$,

$$
\begin{aligned}
d+1+\left\|u_{n}\right\| & \geq J_{\lambda}\left(u_{n}\right)-\frac{1}{\theta} J_{\lambda}^{\prime}\left(u_{n}\right) u_{n} \\
& \geq\left(\frac{m_{0}}{p^{+}}-\frac{m_{1}}{\theta}\right) \int_{\Omega}\left|\Delta u_{n}\right|^{p(x)} d x \\
& +\lambda\left(\frac{1}{\theta\left(q^{+}\right)^{r}}-\frac{1}{\left(q^{-}\right)^{r+1}(r+1)}\right)\left(\int_{\Omega}\left|u_{n}\right|^{q(x)} d x\right)^{r+1} \\
& \geq\left(\frac{m_{0}}{p^{+}}-\frac{m_{1}}{\theta}\right) \| u_{n}||^{p^{-}}
\end{aligned}
$$

This contradicts the fact that $p^{-}>1$. So, the sequence $\left(u_{n}\right)$ is bounded in $X$. By the reflexity of $X$, for a subsequence still denoted $\left(u_{n}\right)$, such that $u_{n} \rightarrow u$ in $X$. From

$$
J_{\lambda}^{\prime}\left(u_{n}\right) \rightarrow 0
$$

we have

$$
J_{\lambda}^{\prime}\left(u_{n}\right)\left(u_{n}-u\right) \rightarrow 0
$$

that is,

$\tilde{M}\left(u_{n}\right) \int_{\Omega}\left|\Delta u_{n}\right|^{p(x)-2} \Delta u_{n} \Delta\left(u_{n}-u\right) d x-\lambda B\left(u_{n}\right) \int_{\Omega}\left|u_{n}\right|^{q(x)-2} u_{n}\left(u_{n}-u\right) d x \rightarrow 0$.

By the Hölder inequality, we obtain

$\left.\left.\left|\int_{\Omega}\right| u_{n}\right|^{q(x)-2} u_{n}\left(u_{n}-u\right) d x\left|\leq \int_{\Omega}\right| u_{n}\right|^{q(x)-1}\left|\left(u_{n}-u\right)\right| d x \leq C\left|u_{n}\right|_{\frac{q(x)}{q(x)-1}}^{q(x)-1}\left|\left(u_{n}-u\right)\right|_{q(x)}$.

Since $q(x)<p^{*}(x)$ for all $x \in \bar{\Omega}$, we deduce that $X$ is compactly embedded in $L^{q(x)}$, hence $\left(u_{n}\right)$ converges strongly to $u$ in $L^{q(x)}$, then

$$
\int_{\Omega}\left|u_{n}\right|^{q(x)-2} u_{n}\left(u_{n}-u\right) d x \rightarrow 0 .
$$

On the other hand, when $\left(u_{n}\right)$ is bounded, there exist positive constants $c_{1}$ and $c_{2}$ such that

$$
c_{1} \leq B\left(u_{n}\right) \leq c_{2} .
$$


So, we have $G\left(u_{n}\right)\left(u_{n}-u\right) \rightarrow 0$. We may assume that, there exist positive constants $c_{3}$ and $c_{4}$ such that

$$
c_{3} \leq \tilde{M}\left(u_{n}\right) \leq c_{4},
$$

we have also

$$
L_{p(x)}\left(u_{n}\right)\left(u_{n}-u\right)=\int_{\Omega}\left|\Delta u_{n}\right|^{p(x)-2} \Delta u_{n} \Delta\left(u_{n}-u\right) d x \rightarrow 0 .
$$

According to the fact that $L_{p(x)}$ satisfies condition $S_{+}$, we have $u_{n} \rightarrow u$ in $X$. The proof is complete.

Proof of theorem 1.1. From lemmas 3.1 and 3.2, we deduce

$$
\max \left(J_{\lambda}(0), J_{\lambda}(e)\right)=J_{\lambda}(0)<\inf _{\|u\|=\rho} J_{\lambda}(u)=\beta .
$$

By lemmas 3.3 and the mountain pass theorem, we deduce the existence of critical points $u$ of $J_{\lambda}$ associated of the critical value given by

$$
c:=\inf _{\gamma \in \Gamma} \sup _{t \in[0.1]} J_{\lambda}(\gamma(t)) \geq \beta
$$

where $\Gamma=\{\gamma \in C([0,1], X): \gamma(0)=0 \gamma(1)=e\}$. This completes the proof.

\section{Proof of Theorem 1.2.}

We start with two auxillary results.

Lemma 3.4. The functional $J_{\lambda}$ is even, bounded from below and satisfies the $(P S)$ condition, $J_{\lambda}(0)=0$.

Proof. It is clear that $J_{\lambda}$ is even and $J_{\lambda}(0)=0$. Recalling that, for all $\lambda>0$, we have

$$
\begin{aligned}
J_{\lambda}(u) & =\tilde{M}\left(\int_{\Omega} \frac{1}{p(x)}|\Delta u|^{p(x)} d x\right)-\frac{\lambda}{r+1}\left[\int_{\Omega} \frac{1}{q(x)}|u|^{q(x)} d x\right]^{r+1} \\
& \geq \frac{m_{0}}{p^{+}} \int_{\Omega}|\Delta u|^{p(x)} d x-\frac{\lambda}{r+1}\left[\int_{\Omega} \frac{1}{q(x)}|u|^{q(x)} d x\right]^{r+1} \\
& \geq \frac{m_{0}}{p^{+}} \int_{\Omega}|\Delta u|^{p(x)} d x-\frac{\lambda}{r+1} \frac{1}{\left(q^{-}\right)^{r+1}}\left[\int_{\Omega}|u|^{q(x)} d x\right]^{r+1}
\end{aligned}
$$

Considering Proposition (2.2),(2.4) and Sobolev immersions, we get 


$$
\begin{aligned}
J_{\lambda}(u) & \geq \frac{m_{0}}{p^{+}} \int_{\Omega}|\Delta u|^{p(x)} d x-\frac{\lambda}{r+1} \frac{1}{\left(q^{-}\right)^{r+1}}\left[\int_{\Omega}|u|^{q(x)} d x\right]^{r+1} \\
& \geq \frac{m_{0}}{p^{+}} \int_{\Omega}|\Delta u|^{p(x)} d x-\frac{\lambda}{r+1} \frac{1}{\left(q^{-}\right)^{r+1}}\left[\max \left\{|u|_{q(x)}^{q^{-}},|u|_{q(x)}^{q^{+}}\right\}\right]^{r+1} \\
& \geq \frac{m_{0}}{p^{+}} \int_{\Omega}|\Delta u|^{p(x)} d x-\frac{\lambda}{r+1} \frac{1}{\left(q^{-}\right)^{r+1}}\left[\max \left\{C^{q^{-}}\left\|\left.u\right|^{q^{-}}, C^{q^{+}}\right\| u \|^{q^{+}}\right\}\right]^{r+1} \\
& \geq \frac{m_{0}}{p^{+}}\left\|u||^{p^{-}}-\frac{\lambda}{r+1} \frac{C^{q^{+}(r+1)}}{\left(q^{-}\right)^{r+1}}\right\| u \|^{q^{+}(r+1)}
\end{aligned}
$$

for all $\|u\|$ large enough. As $p^{-}>q^{+}(r+1), J_{\lambda}$ is bounded from below and coercive become, that is, $J_{\lambda} \rightarrow \infty$ as $\|u\| \rightarrow \infty$.

It remains to show that the functional $J_{\lambda}$ satisfies the (PS) condition to complete the proof. Let $\left(u_{n}\right) \subset X$ be a sequence such that,

$$
J_{\lambda}\left(u_{n}\right) \rightarrow C_{\lambda} \text { and } J_{\lambda}^{\prime}\left(u_{n}\right) \rightarrow 0 \text { in } X^{\prime} .
$$

Then, by the ceorcivity of $J_{\lambda}$, the sequence $\left(u_{n}\right)$ is bounded in $X$, and similar arguments as those used in the proof of lemma 3.3 completes the proof.

Lemma 3.5. For each $n \in \mathbf{N}^{*}$, there exist an $H_{n} \in \Gamma_{n}$ such that

$$
\sup _{u \in H_{n}} J_{\lambda}(u)<0 \text {. }
$$

Proof. Let $v_{1}, v_{2}, v_{3}, \ldots . ., v_{n} \in C_{0}^{\infty}(\Omega)$ such that $\operatorname{supp}\left(v_{i}\right) \cap \operatorname{supp}\left(v_{j}\right) \neq \emptyset$ if $i \neq j$ and meas $\left(\operatorname{supp}\left(v_{j}\right)\right)>0$ for $i, j \in\{1,2,3,4, \ldots, n\}$. Take $F_{n}=$ $\operatorname{span}\left\{v_{1}, v_{2}, v_{3}, \ldots . ., v_{n}\right\}$, it is clear that $\operatorname{dim} F_{n}=n$ and

$$
\int_{\Omega}|v(x)|^{q(x)} d x>0, \text { for all } v \in F_{n} \backslash\{0\} .
$$

Denote $S=\{v \in X:\|v\|=1\}$ and $H_{n}(t)=t\left(S \cap F_{n}\right)$ for $0 \leq t \leq 1$, one has, $t^{q^{+}} \leq t^{q(x)}$ and $t^{p(x)} \leq t^{p^{-}}$, we have

$$
\begin{aligned}
\sup _{u \in H_{n}(t)} J_{\lambda}(u) & \leq \sup _{v \in S \cap F_{n}} J_{\lambda}(t v) \\
& =\sup _{v \in S \cap F_{n}}\left(\tilde{M}\left(\int_{\Omega} \frac{1}{p(x)}|\Delta t v|^{p(x)} d x\right)-\frac{\lambda}{r+1}\left[\int_{\Omega} \frac{1}{q(x)}|t v|^{q(x)} d x\right]^{r+1}\right) \\
& \leq \sup _{v \in S \cap F_{n}}\left(\frac{m_{1} t^{p^{-}}}{p^{-}} \int_{\Omega}|\Delta v|^{p(x)} d x-\frac{\lambda}{r+1} \frac{t^{q^{+}(r+1)}}{\left(q^{+}\right)^{r+1}}\left(\int_{\Omega}|v|^{q(x)} d x\right)^{r+1}\right) \\
& \leq \sup _{v \in S \cap F_{n}}\left(t^{p^{-}}\left(\frac{m_{1}}{p^{-}}-\frac{\lambda}{r+1} \frac{1}{\left(q^{+}\right)^{r+1}} \frac{1}{t^{p^{-}-q^{+}(r+1)}}\left(\int_{\Omega}|v|^{q(x)} d x\right)^{r+1}\right)\right) .
\end{aligned}
$$


Since $m=\min _{v \in S \cap F_{n}} \int_{\Omega}|v|^{q(x)} d x>0$, we may choose $t_{n} \in(0,1]$ which a small enough such that

$$
\frac{m_{1}}{p^{-}}-\frac{\lambda}{r+1} \frac{1}{\left(q^{+}\right)^{r+1}} \frac{1}{t_{n}^{p^{-}-q^{+}(r+1)}} m<0 .
$$

This completes the proof.

Proof of theorem 1.2 From lemmas 3.4, 3.5 and the symmetric mountain pass lemma, we deduce there exists a sequence nontrivial weak solutions $\left(u_{n}\right)_{n}$ such that for any $n$, we have

$$
u_{n}=0, \quad J_{\lambda}^{\prime}\left(u_{n}\right)=0, \quad J_{\lambda}\left(u_{n}\right) \leq 0, \quad u_{n} \rightarrow 0 .
$$

\section{Acknowledgments}

The authors would like to thank the anonymous referees for their clear valuable comments and constructive suggestions.

\section{References}

[1] A. Ayoujil and A.R. El Amrouss, "On the spectrum of a fourth order elliptic equation with variable exponent", Nonlinear analysis: theory, methods \& applications, vol. 71, no. 10, pp. 4916-4926, Nov. 2009, doi: 10.1016/ j.na.2009.03.074

[2] A. Ayoujil and A.R. El Amrouss, "Continuous spectrum of a fourth order nonhomogeneous elliptic equation with variable exponent", Electronical journal differential equation, vol. 2011, Art ID. 24, 2011. [On line]. Available: https:// bit.ly/3nNGV2T

[3] A. Ayoujil, "Existence and nonexistence results for weighted fourth order eigenvalue problems with variable exponent", Boletim da Sociedade Paranaense de Matemática, vol. 37, no. 3, pp. 55-66, Sep. 2019, doi: $10.5269 /$ bspm.v37i3.31657

[4] A. Ayoujil, "Weighted eigenvalue problems involving a fourth-order elliptic equation with variable exponent", International journal of dynamical systems and differential equations, vol. 8, no. 1-2, pp. 66-76, Jan. 2018, doi: 10.1504/ IJDSDE.2018.089096

[5] G. Bin, Q.- M. Zhou and Y.- H Wu, "Eigenvalues of the p (x)-biharmonic operator with indefinite weight", Zeitschrift für angewandte mathematik und physic, vol. 66, no. 3, pp. 1007-1021, Jun. 2015, doi: $10.1007 / \mathrm{s} 00033-014-0465-\mathrm{y}$ 
[6] I. Caffarelli, "Non-local diffusions, drifts and games", in Nonlinear partial differential equations, H. Holden and K. Karlsen, Eds. Berlin: Springer, 2012,pp.pp.37-52, doi: 10.1007/978-3-642-25361-4_3

[7] F. J. S. Corrêa and A. C. dos Reis Costa, "On a bi-nonlocalp(x)-Kirchhoff equation via Krasnoselskiis genus", Mathematical methods in the applied sciences, vol. 38, no. 1, pp. 87-93, Jan. 2014., doi: 10.1002/ mma.3051

[8] F. J. S. Corrêa and A. C. dos Reis Costa, "A variational approach for a bi-nonlocal elliptic problem involving the $\mathrm{p}(\mathrm{x})$-Laplacian and non-linearity with non-standard growth", Glasgow mathematical journal, vol. 56, no. 2, pp. 317-333, Aug. 2013, doi: $10.1017 / \mathrm{s} 001708951300027 \mathrm{x}$

[9] X L. Fan and D. Zhao, "On the spaces $\mathrm{L}^{\mathrm{p}(\mathrm{x})}(\Omega)$ and $\mathrm{W}^{\mathrm{m}, \mathrm{p}(\mathrm{x})}(\Omega)$ ", Journal mathematical analysis applied, vol. 263, no. 2, pp. 424-446, Nov. 2001., doi: 10.1006/ jmaa.2000.7617

[10] X. L. Fan, J. S. Shen and D. Zhao, "Sobolev embedding theorems for spaces $\mathrm{W}^{\mathrm{m}, \mathrm{p}(\mathrm{x})}(\Omega)$ ", Journal mathematical analysis applications, no. 2, pp. 749-760, Oct. 2001, doi: 10.1006/ jmaa.2001.7618

[11] G. Kirchhoff, Mechanik, Leipzig: Teubner, 1883.

[12] R. Kajikiam, "A critical point theorem related to the symmetric mountain pass lemma and its applications to elliptic equations", Journal functional analysis, vol. 225, no. 2, pp. 352-370, Aug. 2005, doi: $10.1016 /$ j.jfa.2005.04.005

[13] T. F. Ma, "Existence results for a model of nonlinear beam on elastic bearings", Applied mathematical letters, vol. 13, no. 5, pp. 11-15, Jul. 2000, doi: 10.1016/S0893-9659(00)00026-4

[14] A. Mao and W. Wang, "Nontrivial solutions of nonlocal fourth order elliptic equation of Kirchhoff type in $\mathbb{R} 3$ ", Journal mathematical analysis applications, vol. 459, no. 1, pp. 556-563, Mar. 2018, doi: $10.1016 /$ j.jmaa.2017.10.020

[15] A. Mao and W. Wang, "Signed and sign-changing solutions of bi-nonlocal fourth order elliptic problem", Journal mathematical physics, vol. 60, Art ID. 051513, May 2019, doi: 10.1063/ 1.5093461

[16] F. Wang, T. An, and Y. An, "Existence of solutions for fourth order elliptic equations of Kirchhoff type on $R^{N^{\prime \prime}}$, Electronic journal of qualitative theory of differential equations, no. 39, pp. 1-11, 2014, doi: 10.14232 / ejqtde.2014.1.39

[17] J. Yaghoub, "Infinitely many solutions for a bi-nonlocal equation with sign-changing weight functions", Bulletin of the Iranian Mathematical Society, vol. 42, no. 3, pp. 611-626, 2016. [On line]. Available: https:/ / bit.ly/3nP0Tuj 
[18] A. Zanga and Y. Fu, "Interpolation inequalities for derivatives in variable exponent Lobegue Sobolev spaces", Nonlinear analysis: theory, methods \& applications, vol. 69, no. 10, pp. 3626-3636, Nov. 2008, doi: 10.1016/ j.na.2007.10.001 